\title{
Towards a dynamical network view of brain ischemia and reperfusion.
}

\author{
Part I: background and preliminaries
}

\author{
Donald J. DeGracia, Ph.D.* \\ Department of Physiology, Wayne State University, Detroit, MI 48201, U.S.A.
}

\begin{abstract}
The general failure of neuroprotectants in clinical trials of ischemic stroke points to the possibility of a fundamental blind spot in the current conception of ischemic brain injury, the "ischemic cascade". This is the first in a series of four papers whose purpose is to work towards a revision of the concept of brain ischemia by applying network concepts to develop a bistable model of brain ischemia. This first paper sets the stage for developing the bistable model of brain ischemia. Necessary background in network theory is introduced using examples from developmental biology which, perhaps surprisingly, can be adapted to brain ischemia with only minor modification. Then, to move towards a network model, we extract two core generalizations about brain ischemia from the mass of empirical data. First, we conclude that all changes induced in the brain by ischemia can be classified as either damage mechanisms that contribute to cell death, or stress responses that contribute to cell survival. Second, we move towards formalizing the idea of the "amount of ischemia", I, as a continuous, nonnegative, monotonically increasing quantity. These two generalizations are necessary precursors to reformulating brain ischemia as a bistable network.
\end{abstract}

Keywords: Boolean networks; brain ischemia and reperfusion; focal cerebral ischemia; global cerebral ischemia; neuroprotection

$\begin{array}{ll}\text { Abbreviations } \\ \text { CBF } & \text { cerebral blood flow } \\ \text { DMSO } & \text { dimethly sulfoxide } \\ \text { GEDI } & \text { gene expression dynamics inspector } \\ \text { MCAO } & \text { middle cerebral artery occlusion } \\ \text { ROS } & \text { reactive oxygen species } \\ \text { STAIR } & \text { stroke therapy academic industry } \\ & \text { roundtable } \\ \text { UPR } & \text { unfolded protein response }\end{array}$

\section{Puzzle pieces}

The general failure of neuroprotection in stroke clinical trials has put the field in a relative state of crisis. But crisis is another word for opportunity and the situation has generated much activity. These activities have largely been directed towards further refinement of existing preclinical and clinical methodology as began by the STAIR group (Gladstone et al. 2002; Dirnagl 2006; Fisher et al. 2005; Fisher et al., 2009), or to further pushing back the empirical frontier (Endres et al 2008). These are clearly important goals. Additional possibilities were offered by O'Collins et al (2006) in their well-known review of neuroprotectants, which sets the stage for the present work:
"No particular drug mechanism distinguished itself on the basis of superior efficacy in animal models of focal ischemia. This may reflect the multifaceted nature of the sequelae of ischemic stroke and suggest a role for combination therapy to target multiple processes. Alternately, it might suggest that our conception of stroke needs reformulation. A tendency to exclusively frame drug activity in terms of the dominant schema of stroke damage (eg. excitotoxicity, free radical damage), coupled with the sometimes arbitrary attribution of a drug mechanism to one of several nonmutually exclusive groupings, might distract from other paradigms with greater explanatory power, thus hindering the development of more effective treatments." (p 474)

Although these statements have generated some controversy, there is prescience to them. While the observation that no neuroprotectant has shown superior efficacy to any other is an important empirical clue we focus on in the $3^{\text {rd }}$ paper of this series, here we highlight the authors' suggestion that we might be looking at the problem incorrectly. Given the urgency of the problem, it is surprising that there has been a relative dearth of effort directed towards the possibility that our conception of stroke may require reformulation. There have been efforts along these lines (Lo

\section{*Correspondence should be sent to:}

Donald J. DeGracia, Ph.D., Department of Physiology, Wayne State University, 4116 Scott Hall, 540 East Canfield Ave., Detroit, Ml 48201, U.S.A. Phone: 313-577-6745; Fax 313-577-5494; E-mail: ddegraci@med.wayne.edu. 
2008; Yenari et al 2008), but these are in the quantitative minority. Further, such efforts tend to be what Huang (2009) has called "metaphorical" as opposed to approaches that are formal or systematic.

More broadly speaking, since stoke is an example of focal brain ischemia, it is natural to expand the conclusion of O'Collins et al to brain ischemia as a whole. Is it possible to reformulate what it means for a brain to experience ischemia? Currently, the idea of the "ischemic cascade" dominates our understanding of the phenomena. To use O'Collins et al.'s term, it is the "dominant schema". But are there alternative possibilities, other "paradigms with greater explanatory power" that may aid us in reformulating our understanding of brain ischemia? In this regard, consider the following statement from Lipton (1999):

"There are three aspects of ischemic cell death which suggest that a formalism that is applied to dynamical systems, in which attractors constitute stable states in a multidimensional space, will provide a useful framework. The first is that there is a very clear insult threshold. ... The second ... is that there is an early profound damage...If the insult is subthreshold, cells recover, whereas if it is superthreshold, they do not.... The third ...is that the end stages of ischemic damage are metastable states that are very different from the normal state of the cell."

"These properties suggest that ischemic cell death can be treated semi-formally, based on the formalism used for describing stable and unstable states of dynamical systems in terms of attractors...in which the emergent properties of networks are manifested."

Lipton's suggestion is clear: use the formalisms applied to dynamical systems as a lens and see what brain ischemia looks like through this lens. Together, the above quotes can be seen as puzzle pieces. If we put these pieces together, a different picture of brain ischemia emerges. The purpose of the present work is to discuss how ideas and methods applied to complex dynamical systems can offer an alternative view of what ischemia does to the brain. We started this project in a previous work (DeGracia 2008) and continue to refine this project in the present series of papers. A complex systems analysis offers an expanded view of brain ischemia, one that envelopes and unifies our current empirical understanding as captured in the term "ischemic cascade," and also offers some surprising implications on many fronts, but especially in terms of therapeutics.

Many interesting ideas will emerge in our discussions below, but one conclusion eclipses all the others and we want to state it up front. The way we here approach, or reformulate, brain ischemia reveals that a mostly implicit assumption appears to dominate the field: the effects of ischemia on the brain are con- ceived of as a linear sum of independent events. In turn, this assumption has tacitly influenced all attempts at neuroprotection. The idea that a system is a linear sum of its component parts is called "superposition", and the associated approach is called "reductionism" (Ahn et al 2006). These are not philosophical, but technical terms. The opposite of superposition and reductionism is that a complex system is greater than the sum of its parts. This is known as "emergence" or "self-organization" and simply results from interactions between the parts of the system. What the study of complex systems teaches in general is that such systems are nonlinear, that superposition does not apply, and that the system components interact in a networked fashion. When the components of a system are networked, they are not independent, as we discuss below.

Therefore, we agree with O'Collins et al (2006) that our conception of stroke, or brain ischemia in general, requires reformulation. We most emphatically suggest that the core of this reformulation should focus on eliminating the (again, mostly implicit) application of the superposition principle to brain ischemia. We shall build a case (specifically in the $3^{\text {rd }}$ paper) that indicates that the failure of neuroprotection is in large measure the failure of the reductionistic approach to the problem. As we go through the exercise of applying complex system concepts, the assumption of linear independence will become obvious. The utility of bringing this assumption to light is that we can begin to consider alternatives, some of which are outlined in the $3^{\text {rd }}$ paper of this series.

\section{Overview of the four papers}

The complexity of our subject matter requires it to be broken out over four separate papers, each building sequentially on the previous. The content and flow of the series is as follows.

The purpose of this, the $1^{\text {st }}$ paper, is two-fold. First, necessary background in network theory is introduced using examples from developmental biology which, perhaps surprisingly, can be adapted to brain ischemia with only minor modification. Then, to move towards a network model, we extract two core generalizations about brain ischemia from the mass of empirical data. These are: (1) all changes induced by ischemia in the brain are either damage mechanisms or stress responses, and (2) we must begin to formalize ischemia as a quantitative variable.

We then carry these generalizations to the $2^{\text {nd }}$ paper and on them begin constructing a network view of brain ischemia. In particular we construct the "postischemic state space", which will be the central construct resulting from an application of network theory to the problem of brain ischemia. However, this will not be a formal model, which would consist of a set of 
equations, because the information is simply not yet there to generate such equations. Instead we move towards this goal by discussing the ingredients, the information, required to get the equations. But in the process, a sufficiently rich picture is built to provide a completely new view of brain ischemia we call the bistable network model of brain ischemia. This bistable model provides a new lens through which the whole field takes on a much different appearance. What appears as complexity through the lens of the "ischemic cascade" is seen in a single unified framework that brings order and simplicity unthinkable from the point of view of the "ischemic cascade".

In the $3^{\text {rd }}$ paper, we compare the bistable model to the "ischemic cascade", where our main focus is on the concept of neuroprotection. The bistable network model offers a radically different concept of neuroprotection. It is in the $3^{\text {rd }}$ paper we tackle the assumption of superposition head-on and discuss how it, more than any single factor, has significantly limited progress in the field. The $3^{\text {rd }}$ paper will perhaps be considered the most controversial.

Finally, the $4^{\text {th }}$ paper wraps up loose ends. We discuss the possible form of the network underlying the post-ischemic state space, and tentatively suggest it may form a small world network architecture. We compare the bistable network model to differential equation-based models of brain ischemia. We make a brief first pass towards applying the bistable model to the problem of focal cerebral ischemia, and get an initial glimpse of the complex networks that operate in the brain after focal ischemia. We end discussing open issues, weakness and points requiring further work.

While here at the beginning we cannot support the claim, by the end of the $4^{\text {th }}$ paper, it will be clear that the bistable network model offers a viable alternative to the "ischemic cascade" as an explanatory framework for brain ischemia. This exercise is neither simple nor superficial. In the four papers we present only the minimum necessary to build a picture that can stand on its own.

\section{Complex systems}

The particular specialties we draw upon as background do not yet have a name that captures all of them under the same umbrella. Terms such as systems biology, dynamic systems theory, chaos theory, nonlinear dynamics, network theory, graph theory each captures different facets, different approaches and techniques. What all of these share in common is they supply tools and concepts by which to understand complex systems and how these change with time. Some examples of success stories where these ideas have furthered the aims of specific areas in biology and medicine include: ventricular fibrillation
(Kaplan and Cohen 1990), transcriptional networks in yeast cells (Futcher 2002), cell differentiation (Huang 2009), cancer (Huang et al 2009), intracellular signaling systems (Ideker 2004), metabolic networks (Yamada and Bork 2009), and in characterizing the relationship between gene mutations and outcome in Alzheimer's disease (Villoslada et al 2009). This seemingly eclectic range of topics illustrates the power and generality of the concepts and tools. It is simply beyond our scope to explain in detail the variety of ideas and techniques that constitute these areas and excellent reviews are available (Burggren and Monticinom 2005; Coveney and Fowler 2005; Villoslada et al 2009). Here, our more circumscribed goal is to introduce essential ideas that we will in turn apply to the problem of brain ischemia. The main ideas we want to explain are Boolean networks with their attractor landscapes, and how these are used to model cell phenotypes.

Before presenting the background information we briefly editorialize to primer the reader's expectations. Sui Huang (2009) has been eloquently vocal about the limits of the dominant way of thinking in which individual molecular species are linked by arrows to other molecular species to describe molecular pathways. There is no question that the intellectual landscape of brain ischemia mostly consists of such pathway diagrams attempting to explain this or that facet of ischemic brain injury. The present author is as guilty as anyone else for contributing these to the field.

Using the "pathways" heuristic causes us to view the workings of the cell as a host of separate molecular pathways occurring in parallel with each other. Indeed, the pathway approach itself is an example of assuming linear independence of the components of which cells are made (Huang 2009). While the pathway approach has produced for us a very detailed understanding of molecular changes in the post-ischemic brain, it nonetheless possesses blind spots that are critically related to the failure of neuroprotection. We know the pathways can and do interact in a variety of fashions, via cross-talk, positive and negative feedback, etc, but the pathway heuristic itself offers no formal means of understanding such interactions. To overcome the limits of the pathway view of cell function, a different approach is needed. Such an approach is provided by network concepts originally developed by Stuart Kauffman (1969). Forty years has seen the network view of complex systems mature to great richness, and many network architectures are now understood (Alon 2007 is one excellent review). We now provide a brief overview of Boolean networks to lay out concepts that will subsequently be applied to the problem of brain ischemia.

\section{Boolean networks}


In a networked view of cell function, the various component parts of the cell, be these proteins, genes, mRNA molecules, signal transduction molecules, etc. are represented as individual nodes in a network. In a Boolean network each node has only two states: on $(=1)$ or off $(=0)$. How can a network whose nodes are either only on or off be biologically relevant? Consider that a node, let's call it "A," represents an enzyme. One may associate the node being "on" $(A=1)$ with the active state of the enzyme, and the node being "off" $(A=0)$ with the inactivate state of the enzyme. Similarly, if a node represents a gene, then the node on would correspond to transcription of the gene, and the node off would mean the gene is not being transcribed.

Interactions of the individual nodes are depicted by links or edges which capture some facet of the relationship between the nodes. In a directed network, the links entering a node are its inputs. The inputs constitute a set of rules to determine the state of a node. This set of rules is technically known as an input function, a concept we focus on in the $2^{\text {nd }}$ paper of this series. Returning to our example enzyme $A$, consider that $A$ has two inputs, call them $B$ and $C$, where $B$ is an activator and $C$ an inhibitor of $A$ (Figure $1)$. If $B$ is active $(=1)$, then $A$ is also active $(=1)$. However, if $C$ is active $(=1)$, then $A$ is inactive $(=0)$. A binary contingency table can be devised that contains all possible combinations of inputs on the node and how these then determine the node's state. The example in Figure 1 shows the output of node $A$ to emulate a Boolean NOT IF operator (Huang and Ingber 2000). Many such network motifs have been identified and shown to emulate specific computa-

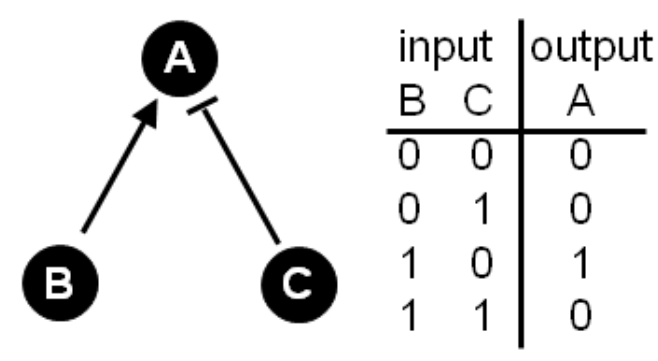

tional operations (Alon 2007).

Figure 1: Example circuit (also called a network motif). Node A might represent an enzyme; nodes $B$ and $C$ could be an activator and inhibitor, respectively, of $A$. The output of $A$ depends upon the inputs $B$ and $C$ as captured in the associated contingency table (right). In this case, the output of A emulates a Boolean NOT IF operator (Huang and Ingber 2000).
Many nodes connected in some particular architecture by many links constitutes the entire network. Within the network many network motifs may be present. In practice, a Boolean network can have thousands of nodes (Yamada and Bork 2009). The state of a given node is determined by its inputs, but these inputs in turn are determined by their own upstream inputs. In this fashion, networks form a kind of closed computational system because of the mutual dependency of the nodes on each others' states. Ultimately, each node in the network is governed by the overall behavior of the network. Thereby individual circuit motifs in the network, each of which may represent a specific pathway, naturally operate in a unified fashion. Thus, network models overcome the limitations of pathway-based models that cannot explicitly account for the dynamics and mutual interactions of the various pathways (motifs) and components (nodes). In a network model, pathway interactions, even distant indirect ones, are implicitly taken into account via the fact that the state of any given node is ultimately a function of the state of every other node.

The state of the all nodes in the network at any instant is described by a state vector (Huang 2009). In a Boolean network of $n$ nodes, the state vector would represent the state of nodes $1,2,3 \ldots n$ as for example $\langle 0,1,0 \ldots 0\rangle$. An alternative state vector might be $<1,1,1 \ldots 1\rangle$. Each state vector is a specific configuration of the network, and the terms "state vector" and "configuration" are used interchangeably. Effectively, a state vector is a point. One can then imagine an n-dimensional space where each point in this space is a specific network state vector. Such a space would then contain all possible configurations of the network. This n-dimensional space is called the state space of the network (Huang 2009). The concept of state space is the central concept we shall use to apply network theory to the problem of brain ischemia.

Not all configurations of the network are equivalent. Configurations are distinguished on the basis of how stable they are. The "stability" of a configuration is a measure of how likely one network configuration is compared to others. The stability of a network configuration derives from the fact that the nodes are linked by formal rules. Consider the following simple example. If node $X$ inhibits node $Y$, but $Y$ also inhibits $X$, then a configuration where both $X$ and $Y$ equal 1 would not be stable, it would be unstable. A stable network configuration would be one in which either $X$ or $Y$ was 0 . Thus the formal rules linking the nodes favor some configurations over others.

When a network assumes an unstable configuration, it will evolve in time though a series of configurations until it achieves a stable configuration. The most -62 - 
stable network configurations are called attractors because the network will naturally be attracted to these states. To indicate the relative stability of configurations, a network state space can be mathematically represented as a landscape of hills and valleys. The higher a configuration is on a hill, the more unstable it is. The deeper into a valley, the more sta- ble the configuration. The lowest point of a valley is the most stable and constitutes an attractor state of the network (see Figure 2 caption for additional details). If the network configuration is unstable (on a hill) it will change by moving over the state space landscape until it encounters a stable attractor state.

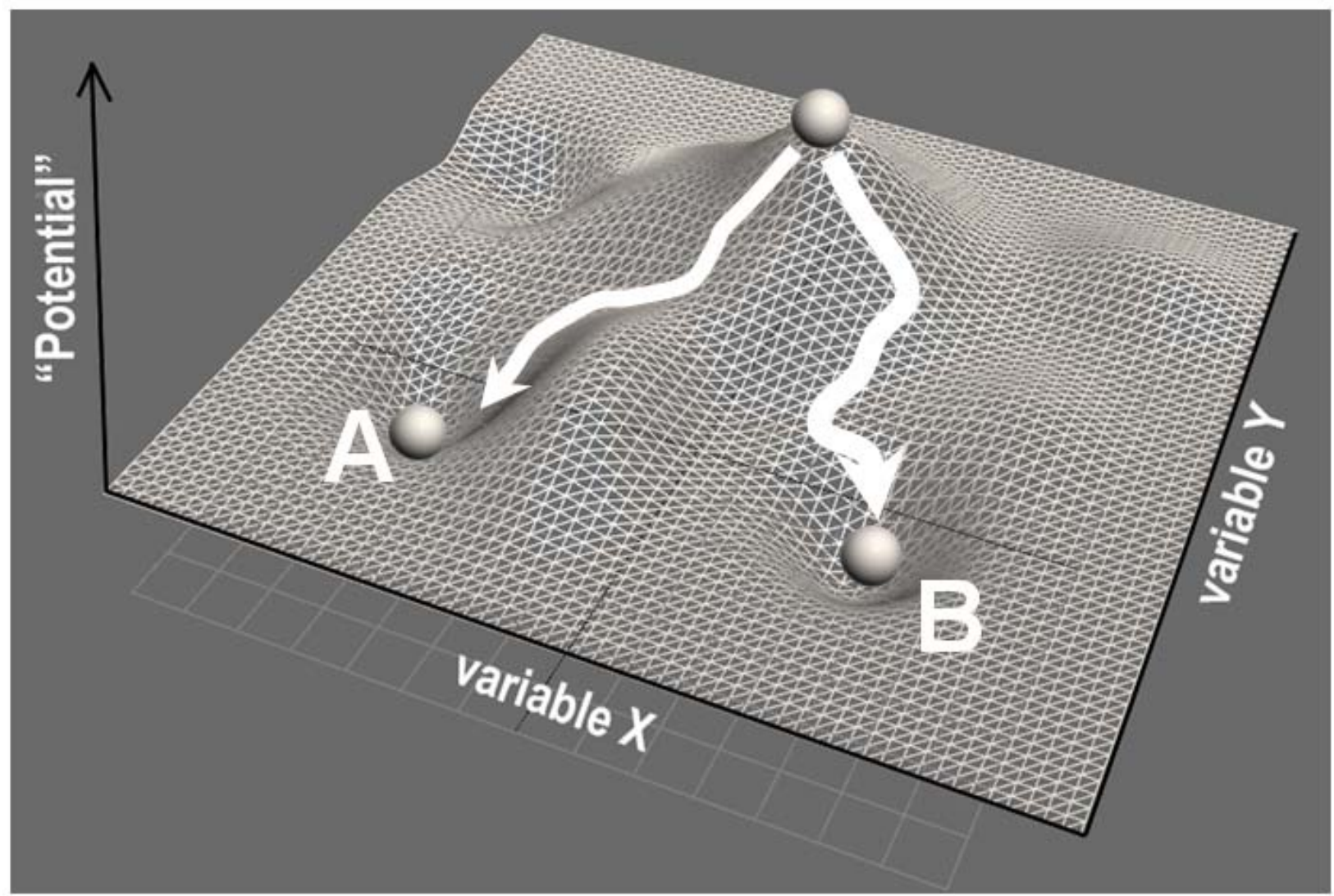

Figure 2: A network state space. In general, network state spaces are n-dimensional spaces where each point in the space represents a unique network configuration of $\mathrm{n}$ nodes. This image shows a 2dimensional network to allow visualization of the state space. This example is not a Boolean network, but is dependent on the continuous variables $x$ and $y$. A value can be calculated that represents the stability of a point (e.g. a network configuration) in state space and this is shown here as the $3^{\text {rd }}$ dimension of the graph. The third dimension acts like a potential function (e.g. higher energy states are more unstable, lower energy states are more stable), but for technical reasons is not a true potential and is therefore called a "quasipotential" (Huang 2009). When the state space is plotted as a function of the quasi-potential, the state space takes on the form of a landscape containing hills and valleys. The hills are unstable and the valleys are stable configurations of the network. The network configuration is "attracted" to the valleys, and hence the valleys are called "attractors". In this image, two different attractors A and B are depicted. The network configuration (shown as a marble) is at the top of the hill and can move in time through a series of network configurations depicted by the white arrows. The path through state space is called a trajectory. Depending on network parameters, the state of the network may take different trajectories towards different attractors.

It is important to emphasize to the reader that we are only outlining the mathematics behind these concepts in a cursory fashion. Further detail would take us beyond our intended scope. The ideas are presented at a high conceptual level which is suitable for our present purposes. But it is critical that the reader keep in mind that these are not metaphorical concepts, but are actual forms of mathematical representation of complex systems. For those interested, additional detail can be found in the citations.

\section{Differentiation and state spaces}


While seemingly abstract, it is critical to note these ideas have been successfully applied to real empirical issues. We now review how these ideas have been applied in the field of developmental biology to model the process of cell differentiation. The example is instructive and sets a precedent for applying the ideas to brain ischemia, not just by way of analogy, but because the same networks used to model differentiation can be applied, with some modification, to the problem of brain ischemia.

In studies of cell differentiation, Boolean networks have been used to represent cellular genotypes (Huang and Ingar 2000; Huang et al 2005; Huang 2009). Different network configurations correspond to different patterns of genes being either on or off, that is, to different patterns of gene expression or different transcriptomes. Specific network configurations (i.e. patterns of gene expression) in turn underlie different phenotypes. Therefore, the network state space is taken to contain formal representations of the possible phenotypes of the stem cell, including the stem cell phenotype and any possible phenotypes into which it may differentiate. Each phenotype is represented in the state space as an attractor (e.g. a stable pattern of gene expression). The process of differentiation is then conceptualized as the change of the state vector from an attractor state that represents the progenitor cell (and its corresponding pattern of gene expression) to an attractor state that represents the differentiated cell (and its corresponding pattern of gene expression) (Figure 3).

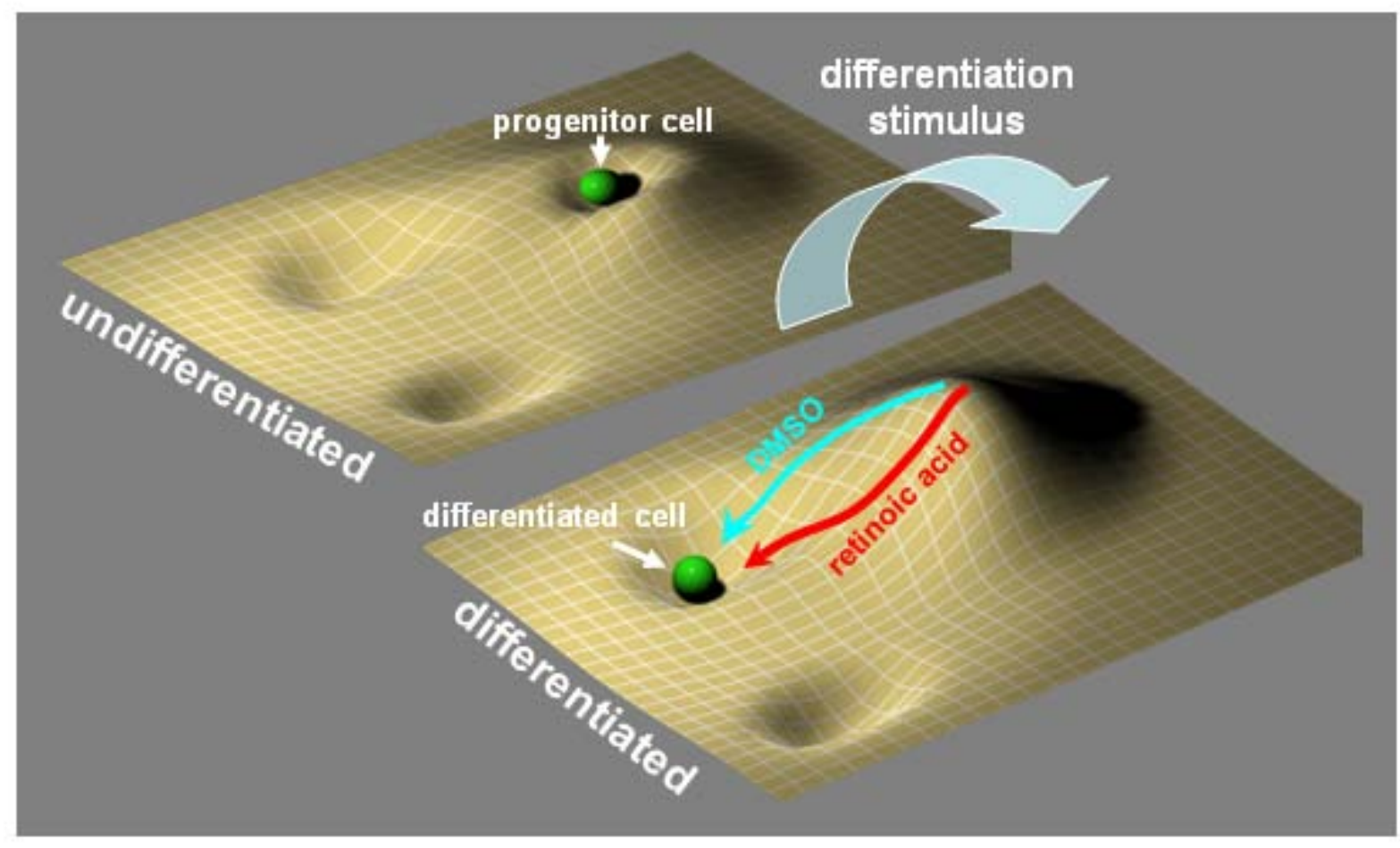

Figure 3: Differentiation induces a bifurcation in the network state space, qualitatively changing the shape of state space. Here, the depression at the top of the hill is the attractor for the undifferentiated cell phenotype. This attractor is lost after cells are exposed to an inducer of differentiation (DMSO or retinoic acid in the case of HL60 cells). The shape change in state space provides a "motive force" for the cell transcriptome (depicted by the green marble) to move through state space into one of the two possible differentiated attractor states depicted here. Different inducers may trigger different trajectories (DMSO, blue line; retinoic acid, red line) that nonetheless converge to the same final phenotype. Image is loosely adapted from data in Huang et al 2005.

The movement of the state vector from the progenitor attractor to the differentiated cell attractor is the trajectory of the cell as it differentiates (Figure 3). The network transforms through a series of configurations (i.e. patterns of gene expression), tracing out a path, the trajectory, on the landscape of hills and valleys of state space. Such movement through state space provides the dynamic element to network models.

It is of interest to discuss how these concepts are used experimentally. Using microarray data, Huang et al (2005) have elegantly shown how different differentiation signals can induce different trajectories in state space that nonetheless lead to the same final -64 -

J Exp Stroke Transl Med (2010) 3(1): 59-71

Society for Experimental Stroke (www.s4es.org) 
phenotype. Here, HL60 cells were differentiated into neutrophils using either DMSO or retinoic acid. Microarrays of 2773 transcripts were taken along a 168 hr time course after induction of differentiation. Using GEDI analysis (a method for comparing relative gene expression in the microarrays, Eichler et al 2003), it was shown that each stimulus caused divergent patterns of gene expression early in the differentiation time course, but these eventually converged to the same pattern of gene expression when the cells had attained their final differentiated phenotypes. These data were then interpreted to indicate that each stimulus triggered differentiation along a different trajectory in state space, but the trajectories converged when the cells entered the attractor state of the final differentiated phenotype. Thus, the time course of changes in gene expression, accessible by microarrays, provided an experimental marker for the trajectory through the genomic state space.

In a more comprehensive analysis of the differentiation trajectory, Huang et al (2007) showed how a differentiating signal severed first to destabilize the attractor state of the progenitor cell. Application of a differentiation inducer served to convert the shallow valley of the progenitor attractor state into an unstable hill, from which the cells (the network) naturally "flowed" towards the attractor state of the final differentiated phenotype (Figure 3). The qualitative change in the shape of state space was due to a $b i-$ furcation in certain parameters underlying the network model.

Summary. This wraps up our brief review of those elements of networks that will prove useful to us ahead. To summarize, the core ideas we extract from the above are:

1. A cell phenotype can be formally described as an attractor state in a network state space.

2. The process of a cell changing its phenotype can be modeled as a change from one attractor to another attractor along a trajectory on the state space landscape.

3. Some type of perturbation or initiating circumstance can provide the "motive force" for a cell type to move through the state space landscape.

It is not surprising that these concepts have derived from the study of development and differentiation where changes in cell phenotypes are the focus of study. Similar ideas are being applied in the fields of evolution (Steward 2003) and cancer (Huang et al 2009) where again, changes in phenotypes are central to the respective fields. The application of these ideas to brain ischemia is predicated on the recognition that ischemia changes the phenotype of brain cells. What post-ischemic cells have in common with differentiating or transformed cells is that they reprogram their gene expression (DeGracia et al 2008). The genetic reprogramming of post-ischemic brain cells is the basis by which we can recognize that these cells indeed undergo phenotypic changes, opening the door to modeling ischemia with the concepts discussed above.

\section{Conceptualizing ischemia: damage mechan- isms and stress repsonses}

Before presenting our discussion of brain ischemia as a network phenomenon in the $2^{\text {nd }}$ paper, we need to clarify concepts about brain ischemia to make them amenable to the type of analysis discussed above. All of us in the field know the story of the ischemic cascade (e.g. as recently summarized by Brouns and De Deyn, 2009; or see Figure 1 of Lipton, 1999) so we won't belabor the point here. What we are aiming for here is try to classify our understanding of the elements that make up the ischemic cascade. Further, we want to do this in such a fashion as to keep the goal of therapeutics within a short intellectual reach.

In the simplest analysis, the elements of the ischemic cascade consist of a set of reactions or pathways that damage brain cells. Examples of such elements include calcium ion overload, excitotoxicity, production of reactive oxygen species (ROS), lipid peroxidation, proteolysis, and the list goes on and on. Every one of these elements follow in a primary, secondary, tertiary, etc. fashion from the reduction or loss of ATP (Lipton 1999). Loss of ATP is the most proximal event following a reduction or cessation of blood flow. Thereby, when cell death occurs, we attribute its causation to some combination of these damage mechanisms. We emphasize this term because it will be a central concept ahead. Indeed, all neuroprotective attempts have been directed to halt one or another of these damage mechanisms, of which we say more in the $3^{\text {rd }}$ paper of this series.

However, the case is not so simple. All cells, including those of the brain, have evolved means for coping with damaging stimuli, collectively called stress response pathways. We also emphasize this term as it too will figure importantly ahead. A number of these have been identified in general, and are known to be activated following brain ischemia and reperfusion in particular. These will not be discussed in detail here as that would take us beyond our intended scope. Some classical examples of ischemia-induced stress responses include the heat shock response (Nowak 1990; Sharp et al. 1999), endoplasmic reticulum stress pathways (Paschen 1996; DeGracia et al. 2002), DNA repair pathways (Ferrer and Planas 2003; Strosznajder et al. 2005), proteosome activation 
(Meller 2009), and the pro-survival response centered on the kinase Akt (Zhao et al. 2006). We previously discussed ways to conceptualize the relationship between damaging stimuli and stress responses in the post-ischemic brain (DeGracia, 2008).

There seems to be a general under-appreciation that the "ischemic cascade" consists not only of damage mechanisms, but also of stress responses (Endres et al 2008; Lo 2008). Our lab has been studying a facet of the post-ischemic stress response for years, but only slowly has this become clear. The inhibition of protein synthesis that occurs in post-ischemic neurons was initially thought of as a form of cell damage, one that reversibly goes away in cells that survive, but persists in cells that will die (White et al 2000). However, work over the past couple decades from a number of labs has now made clear that postischemic inhibition of protein synthesis is a marker of the induction of post-ischemic stress responses (Paschen 1996; Martin de la Vega et al., 2001; DeGracia et al, 2008). Importantly, we now know that the induction of post-ischemic stress responses is a clear-cut marker of the genetic reprogramming of post-ischemic brain cells (DeGracia et al., 2008).

Slowly the role of stress responses is gaining appreciation, but how they fit in is still open to question. Eng Lo (2008), for example, recently wrote about the biphasic nature of post-ischemic responses where an initially damaging response may subsequently be involved in repair. We appreciate this viewpoint because several years ago we proposed a related type of role for the expression of the unfolded protein response (UPR) in post-ischemic neurons (DeGracia et al., 2002). The UPR though is not so much biphasic as it is "two faced:" it can lead either to cell repair or cell death depending on the extent of damage to the endoplasmic reticulum (DeGracia et al., 2002).

More generally, there is clearly an important interplay of damage and repair reactions occurring in postischemic cells. This is important from the perspective of neuroprotection; instead of just inhibiting damage mechanism, we also have the option of enhancing post-ischemic stress responses and repair mechanisms, something we (DeGracia, 2008) and many others (Dirnagl and Meisel 2008; Gutiérrez et al 2009) have discussed in some detail and therefore won't dwell on here. Given the potential for serious clinical application, there is a real need to systematically understanding not just the role of damage pathways, but also the role of stress responses in the postischemic brain. We shall see that the network view presented here provides exactly such a systematic approach.

\section{Taming the complexity of the post-ischemic brain}

To begin to move in such a systematic direction, we suggest that each element of the "ischemic cascade" can be classified as either a damage mechanism or a stress response. Or more broadly, let us dispense with using the idea of "ischemic cascade" as a middle man, and just simply say that all the changes induced in the brain by ischemia can be classified as belonging either to the class of damage mechanisms or the class of stress responses. This, we suggest, is the first important generalization required to reformulate the problem of brain ischemia as a whole.

Having made this generalization, let's consider what is perhaps its most important implication. The damage mechanisms and stress responses will be mutually antagonistic. While damage mechanisms activate stress responses, the purpose of the stress responses is to get rid of the damage mechanisms (DeGracia, 2008). Stress responses will inhibit damage mechanisms (and also induce cellular repair, which is a downstream aspect of most stress responses). However, while the damage mechanisms serve to induce stress responses, they are also antithetical to them. If the damage is intense enough, it can also damage the mediators of the stress responses. Clearly damage mechanisms are not directed towards particular functional ends, but represent entropic processes that can potentially, but incidentally, destroy mediators of stress responses as well as any other organized aspect of cell function. The interference of stress responses by damage mechanisms is one fashion by which a seemingly good thing, a stress response, can transform into a bad thing along the lines that Eng Lo suggests. The key point is that, once turned on, the net functional outcome is that the damage mechanisms and stress responses compete with each other. Again, they are mutually antagonistic. But how do we get a handle on this mutual antagonism? That was alluded to with the word "intensity", which we now discuss.

\section{Thresholds}

Our second important generalization is not only aimed at addressing how to understand the mutual antagonism of damage mechanisms and stress responses, it also relates to the fact that ischemia does not always kill. In fact, ischemia can be "good" if it is causes the tissue to become preconditioned (Kirino 2002). The underlying point is that ischemia can be more or less. In some sense it is a quantitative variable. It has long been recognized that there are many thresholds related to the amount of ischemia the brain experiences. The idea of "thresholds" is intimately related to the idea of the amount of ischemia, so let's briefly discuss the use to which the word "threshold" has been used in the field. 
The initial use of the idea of an ischemic threshold was to distinguish loss of brain electrical activity from loss of cell membrane functional integrity at different blood flow decrements (Opitz and Schneider 1950; Symon et al. 1977; Astrup et al. 1981). Hossmann's group refined this notion by defining viability thresholds in which specific well-defined metabolic or electrical (functional) events occurred at specific values of blood flow decrement (Hossmann 2006).
Some of the viability thresholds are summarized in Table 1. More recently, the concept of viability thresholds has had its meaning altered where it is used to correlate neuroimaging parameters with different post-stroke outcomes (Warach 2001). In general, the use to which the concept of "threshold" is put in these cases is phenomenological: at some specific decrement of cerebral blood flow (CBF), a phenomenon $X$ occurs.

Table 1: Viability thresholds in terms of blood flow rate (in $\mathrm{mL} \mathrm{g}^{-1} \mathrm{~min}^{-1}$ ). Adapted from Hossmann (2006).

\begin{tabular}{llll}
\hline Metabolic Thresholds & \multicolumn{3}{l}{ Electrical \& Functional thresholds } \\
\hline protein synthesis inhibited & $<0.55$ & decline of EEG & $0.23-0.25$ \\
increased glucose utilization & $<0.35$ & reversible hemiparalysis & 0.23 \\
acidosis & $<0.26$ & neurotransmitter release & 0.20 \\
decreased glucose utilization & $<0.25$ & loss of spontaneous activity & 0.18 \\
ATP decline & $0.10-.20$ & irreversible paralysis & $<0.17-0.18$ \\
brain edema & 0.1 & anoxic depolarization & $<0.15$ \\
\hline
\end{tabular}

When we consider Peter Lipton's quote at the beginning of this paper, he also utilizes the idea of threshold, but in a different sense than those used in the previous paragraph. Lipton's use of the term is more akin to a concept that has been floating around the field but which has not made it into print, and that is the concept of threshold as captured in Tadeusz Wieloch's "sandwich model" of ischemic cell death (Tadeusz Wieloch, personal communication). As Wie-

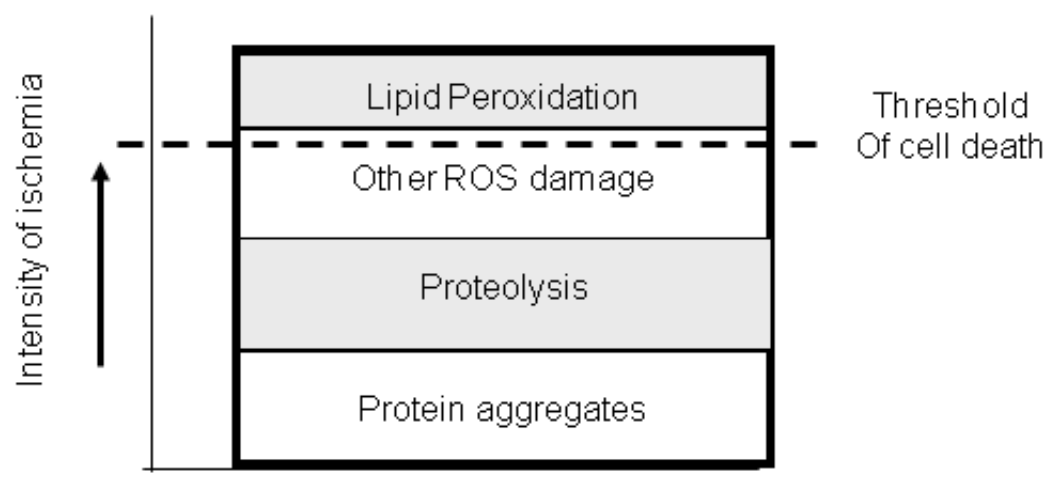

loch's sandwich model serves an important purpose in this presentation, we now outline his idea. Wieloch's "sandwich model" is a sandwich because one envisions stacking all the damage mechanisms. The height of each damage mechanism reflects its intensity. If the total height of the sandwich passes a critical threshold, the cell dies. The "sandwich model" is illustrated in Figure 4.

Figure 4: Illustration of Tadeusz Wieloch's "sandwich model" of brain ischemia and reperfusion injury. The various damage mechanisms are stacked to make a "sandwich". The height of any one damage mechanism reflects the intensity of that form of damage. Together, the different forms of damage add up. If their sum exceeds a critical threshold, the cell dies. Presumably, the dependent variable that sets the height of each damage mechanism, and therefore their summed intensities, is the total amount of ischemia perienced by the brain. Note that we have only arbitrarily included four damage mechanisms. Many more have been identified and the sandwich would in reality have many, many layers.

Lipton and Wieloch share a similar idea of threshold, and their use of the term is akin to the idea of bifurcation as described above: a parameter passes a critical threshold value, causing a qualitative change to the system as a whole. The phrase "system as a whole" is what distinguishes the Lipton/Wieloch usage from the phenomenological usage of Astrup or
Hossmann. The entire system transforms on the other side of a Lipton/Wieloch threshold.

Now, to what is the term "threshold" applied when used in the Lipton/Wieloch sense? Lipton specifically says "insult threshold." The insult under consideration is ischemia. Therefore we can say that Lipton is 
referring specifically to the amount of ischemia. In the case of the sandwich model, we can perhaps safely infer that Dr. Wieloch is also referring to the amount of ischemia insofar as the amount of ischemia will set the intensity of the ensuing damage mechanisms. Therefore, both Lipton and Wieloch are attempting to formalize what is well-known to all of us: if the cells experience some specific amount of ischemia, they will die.

\section{The amount of ischemia}

We are therefore naturally led to ask just what exactly is meant when we say the "amount of ischemia?" This is a central concept in the present work, and we admit that we will now gloss over the topic because it is in fact very complex. The complexity revolves around the fact that the term "ischemia" refers to a range of blood flow decrements, including zero blood flow. The former is called "incomplete" ischemia, the later "complete" ischemia. The term "incomplete ischemia" has the quality of "bigger than a bread basket, but smaller than a car." The term incomplete ischemia comes into play when the term "hypoperfusion" is not strong enough to accurately describe the effect of the blood flow decrement, setting the top end of the range. The range then extends down to some differential that is greater than zero blood flow (e.g. "trickle flow"; Hossmann 2006). In addition, ischemia can be either "focal" or "global", adding additional dimensions of complexity to the issue. We would suggest that application of the term "ischemia" to such a wide range of blood flow conditions has made it difficult to get a precise handle on the whole phenomena. What we now gloss over is the task of trying to get a handle on thinking of ischemia as a quantitative variable.

In some sense, the "amount" of ischemia is the product of the decrement in blood flow and the duration for which this occurs. That is, the "amount" of ischemia is the answer to an integral of the blood flow decrement from time zero to the time that marks the end of the ischemia. Mathematically, we can make a first stab at defining the amount of ischemia, $I$, as follows:

$$
I=\int_{t 1}^{t 2} \Delta C B F \bullet d t
$$

Where:

$\triangle C B F=$ the decrement in $\mathrm{CBF}$.

$d t=t_{2}-t_{1}=$ the duration of the CBF decrement.

If $I$ is expressed as the CBF decrement, then $I=0$ at $100 \%$ CBF and is nonzero at CBF $<100 \%$. While we can imagine virtually any pattern of CBF decre- ment as a function of time, Figure 5 presents a simple illustration of the concept. Here two equal "amounts" of incomplete ischemia are shown. For simplicity sake, we assume these are global ischemia. In the first case, CBF is reduced to $20 \%$ (creating an ischemia of $80 \%$ ) for 15 min then returned back to $100 \%$ (red curve). In the second case, CBF is reduced to $80 \%$ (ischemia of $20 \%$ ) for 60 min before being returned to $100 \%$ (green curve). Integrating the area under each curve to get $I$ shows the two curves result in equal values of $I$, as illustrated by the $X s$ marking the respective areas.

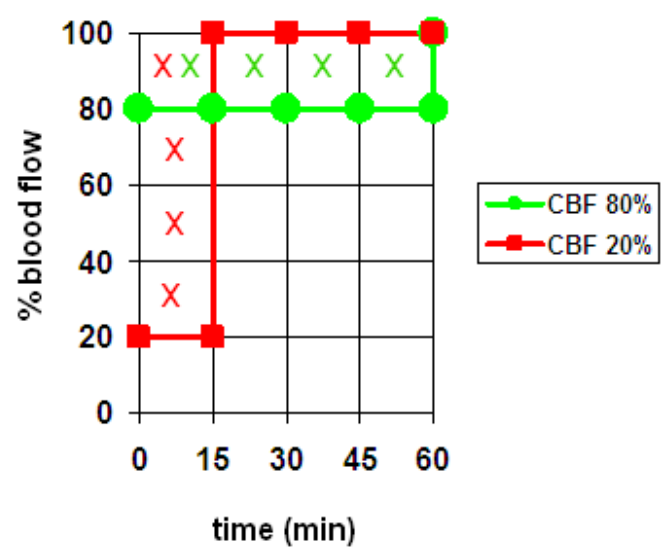

Figure 5: Simple graphs of two different conditions of incomplete global cerebral ischemia caused by differentially reducing cerebral blood flow (CBF) to different decrements for different durations. CBF at $20 \%$ for 15 min gives the same area under the curve as $80 \%$ CBF for 60 min. However, it is well known that these two forms of ischemia lead to very different outcomes.

However, because of the phenomenological thresholds shown in Table 1, we know that these two conditions are totally different from a functional point of view. A $20 \%$ reduction in brain blood flow for 60 min is more a hypoperfusion than an incomplete ischemia, and one isolated instance of this would not have any adverse effect on long-term brain function. On the other hand, one instance of $15 \mathrm{~min}$ of $20 \%$ blood flow will certainly disrupt the brain during the ischemic period and perhaps be on the cusp of inducing neuronal death. The point here is that just because we can imagine a mathematically valid concept of $I$ does not mean it is realistic. The examples in Figure 5 may be mathematically equal but they are not functionally comparable.

One obvious generalization follows from these considerations: To compare different amounts of ischemia, $I$, the ischemia must occur at the same absolute level of CBF. Then, different durations would be proportional. This generalization follows from the phenomenological viability thresholds (Table 1 ) and illu-68 - 
strates how they must play a central role in any conception of the "amount" of ischemia. Further, the idea that different durations of a given CBF decrement are proportional really only applies to global ischemia where the entire brain is exposed to the same CBF decrement (with caveats discussed in the next paper).

When we consider focal ischemia the whole picture gets much more complex. First, focal ischemia potentially encompasses the entire blood flow range: CBF can be $0 \%$ at the site of obstruction and will be $100 \%$ sufficiently far from the obstruction. Second, the blood flow gradient away from the site of a focal obstruction will have a complex shape that is a function of the shape of the obstruction and where in the vasculature the obstruction occurs (Hademenos and Massoud 1997). That is, spatial factors must also be taken into consideration when defining I for any focal case. In this regard, the blood flow gradient is not even expected to be spatially continuous from $0 \%$ to $100 \%$ because blood flows in a three dimensional tree structure in the brain, which is expected to generate a spatial distribution with discontinuities in it. Coupling this complexity with individual variations in the cerebral vasculature, there is no a priori method to determine the gradient of CBF around any arbitrary focal insult, a practical issue faced daily by neurologists treating stroke patients.

However, in principal such a gradient exists. Mathematical models of focal ischemia provide a means to construct a blood flow gradient (Hudetz et al 1982). What is relevant for the present discussion is to recognize that, unlike global ischemia, focal ischemia causes different levels of CBF to occur simultaneously in the same brain. Even with our knowledge of phenomenological thresholds (Table 1), it is clear that focal ischemia will induce much more complex changes in the brain because of the coexistence of different levels of CBF in the same brain. While these considerations may seem trivial to experts in stroke, we return to consider them in the $4^{\text {th }}$ paper, after developing our bistable model of brain ischemia. We will see that focal ischemia appears in a different light using this lens.

Thus, the bottom line to this discussion is that to meaningfully discuss ischemic thresholds in the Lipton/Wieloch sense, we are forced to consider only global ischemia in the present work. Then, different amounts of ischemia, $I$, are synonymous with different durations of ischemia at some particular CBF level. For the present discussion we choose CBF to be zero, or the CBF decrement to be $100 \%$. That is, the following discussions are only meant to apply to complete global ischemia, in part because there is a lot of literature we can then draw upon.
We could have stated this outright without going through the above discussion. But the purpose of the above is to highlight the need to seriously consider the issue and work towards some systematic means of saying "the amount of ischemia." For example, how does 2 hours of middle cerebral artery occlusion (MCAO) ischemia compare to 10 min of global cerebral ischemia? There is at present no precise answer to this question. The above considerations move us in a direction where such a question may be meaningfully considered. The necessity to have a means by which to determine "the amount of ischemia" will become clear as we progress with the discussion.

\section{Summary}

So, to summarize our generalizations to this point, which are two:

1. All changes induced in the brain by ischemia are either damage mechanisms or stress responses and these are mutually antagonistic.

2. Within the constraints discussed above, ischemia can be viewed as a nonnegative quantitative variable, $I$, that increases continuously and monotonically as a function of time.

Therefore, we can assess the relative roles of the damage mechanisms and stress responses induced by ischemia against a scale of the amount of ischemia, $I$, applied to the brain. These two generalizations provide us the main tools we need to begin to think about a network view of brain ischemia, which we take up in the $2^{\text {nd }}$ paper of this series.

\section{Acknowledgement}

The full acknowledgements are presented in the $4^{\text {th }}$ paper of this series. This work was supported by the National Institute of Neurological Disorders and Stroke (NS-057167).

\section{Conflict of interest}

None

\section{References}

Ahn AC, Tewari M, Poon C-S, Phillips RS. (2006) The Limits of Reductionism in Medicine: Could Systems Biology Offer an Alternative? PLoS Med 3: 709-713

Alon U. (2007) Network motifs: theory and experimental approaches. Nat Rev Genet 8:450-461

Astrup J, Symon L, and Siesjo BK. (1981). Thresholds in cerebral ischemia-The ischemic penumbra. Stroke $12: 723-725$ 
Brouns R, De Deyn PP. (2009) The complexity of neurobiological processes in acute ischemic stroke. Clin Neurol Neurosurg 111:483-495

Burggren WW, Monticinom MG. (2005) Assessing physiological complexity. J Expt Bio 208: 3221-3232

Coveney PV, Fowler PW. (2005) Modelling biological complexity: a physical scientist's perspective. J R Soc Interface 2:267-280

DeGracia DJ, Jamison JT, Szymanski JJ, Lewis MK. (2008) Translation arrest and ribonomics in post-ischemic brain: layers and layers of players. $J$ Neurochem 106:2288-2301

DeGracia DJ, Kumar R, Owen CR, Krause GS, White BC. (2002) Molecular pathways of protein synthesis inhibition during brain reperfusion: implications for neuronal survival or death. J Cereb Blood Flow Metab 22:127141

DeGracia DJ. (2008) Ischemic damage and neuronal stress responses: Towards a systematic approach with implications for therapeutic treatments. In New Frontiers in Neurological Research. Wang DQ and Ying W (eds). Research Signpost,Kerala, India.. pp 235-264

Dirnagl U. (2006) Bench to bedside: the quest for quality in experimental stroke research. J Cereb Blood Flow Metab 26:1465-1478

Dirnagl U, Meisel A. (2008) Endogenous neuroprotection: Mitochondria as gateways to cerebral preconditioning? Neuropharmacology 55:334-344

Endres M, Engelhardt B, Koistinaho J, Lindvall O, Meairs S, Mohr JP, Planas A, Rothwell N, Schwaninger M, Schwab ME, Vivien D, Wieloch T, Dirnagl U. (2008) Improving outcome after stroke: overcoming the translational roadblock. Cerebrovasc Dis 25:268-278

Ferrer I, Planas AM. (2003) Signaling of cell death and cell survival following focal cerebral ischemia: life and death struggle in the penumbra. J Neuropathol Exp Neurol 62 :329-339

Fisher M, (Stroke Therapy Academic Industry Roundtable, I. V.). (2005) Enhancing the Development and Approval of Acute Stroke Therapies: Stroke Therapy Academic Industry Roundtable. Stroke 36:1808-1813

Fisher M, Feuerstein G, Howells DW, Hurn PD, Kent TA, Savitz SI, Lo EH. (2009) Update of the stroke therapy academic industry roundtable preclinical recommendations. Stroke 40:2244-2250

Futcher B. (2002) Transcriptional regulatory networks and the yeast cell cycle. Curr Opin Cell Biol 14:676-683

Eichler GS, Huang S, Ingber DE. (2003) Gene Expression Dynamics Inspector (GEDI): for integrative analysis of expression profiles. Bioinformatics 19:2321-2322

Gladstone DJ, Black SE, Hakim AM. (2002) Toward wisdom from failure: lessons from neuroprotective stroke trials and new therapeutic directions. Stroke 33:21232136
Gutiérrez M, Merino JJ, de Leciñana MA, Díez-Tejedor E. (2009) Cerebral protection, brain repair, plasticity and cell therapy in ischemic stroke. Cerebrovasc Dis 27(Suppl 1):177-186

Hademenos GJ, Massoud TF. (1997) Biophysical mechanisms of stroke. Stroke 28:2067-2077

Hossmann KA. (2006) Pathophysiology and Therapy of Experimental Stroke Cellular and Molecular Neurobiology 26:1057-1083

Huang S, Eichler G, Bar-Yam Y, Ingber DE. (2005) Cell fates as high-dimensional attractor states of a complex gene regulatory network. Phys Rev Lett 94:128701-1128701-4

Huang S, Ernberg I, Kauffman S. (2009) Cancer attractors: a systems view of tumors from a gene network dynamics and developmental perspective. Semin Cell Dev Biol 20:869-876

Huang S, Guo YP, May G, Enver T. (2007) Bifurcation dynamics in lineage-commitment in bipotent progenitor cells. Dev Biol 305:695-713

Huang S, Ingber DE. (2000) Shape-dependent control of cell growth, differentiation, and apoptosis: switching between attractors in cell regulatory networks. Exp Cell Res 261:91-103

Huang S. (2009) Reprogramming cell fates: reconciling rarity with robustness. BioEssays 31:546-560

Hudetz AG, Halsey JH, Horton CR, Conger KA, Reneau DD. (1982) Mathematical simulation of cerebral blood flow in focal ischemia. Stroke 13:693-700

Ideker T. (2004) A systems approach to discovering signaling and regulatory pathways--or, how to digest large interaction networks into relevant pieces. Adv Exp Med Biol 547:21-30

Kaplan DT, Cohen RJ. (1990) Searching for chaos in fibrillation. Ann N Y Acad Sci 591:367-374

Kauffman S. (1969) Homeostasis and differentiation in random genetic control networks. Nature 224:177-178.

Kirino T. (2002) Ischemic tolerance. J Cereb Blood Flow Metab $22: 1283-1296$

Lipton P. (1999) Ischemic cell death in brain neurons. Physiol Rev 79:1431-1568

Lo EH. (2008) A new penumbra: transitioning from injury into repair after stroke. Nat Med 14:497-500

Martín de la Vega C, Burda J, Nemethova M, Quevedo C, Alcázar A, Martín ME, Danielisova V, Fando JL, Salinas M (2001) Possible mechanisms involved in the down-regulation of translation during transient global ischaemia in the rat brain. Biochem J 357:819-826

Meller R. (2009) The role of the ubiquitin proteasome system in ischemia and ischemic tolerance. Neuroscientist 15:243-260

Nowak TS Jr. (1990) Protein synthesis and the heart shock/stress response after ischemia. Cerebrovasc Brain Metab Rev 2:345-366 
O'Collins VE, Macleod MR, Donnan GA, Horky LL, van der Worp BH, Howells DW. (2006) 1,026 experimental treatments in acute stroke. Ann Neurol 59:467-477

Opitz E, Schneider M. (1950). U“ ber die Sauerstoffversorgung des Gehirns und den Mechanismus der Mangelwirkungen. Ergebn Physio. 46:126-260

Paschen W. (1996) Disturbances of calcium homeostasis within the endoplasmic reticulum may contribute to the development of ischemic-cell damage. Med Hypotheses 47:283-288

Sharp FR, Massa SM, Swanson RA. (1999) Heat-shock protein protection. Trends Neurosci 22:97-99

Steward I. (2003) Self-organization in evolution: a mathematical perspective. Phil Trans R Soc Lond 361:11011123

Strosznajder RP, Jesko H, Zambrzycka A. (2005) Poly(ADP-ribose) polymerase: the nuclear target in signal transduction and its role in brain ischemiareperfusion injury. Mol Neurobiol 31:149-167

Symon L, Branston NM, Strong AJ, Hope TD. (1977). The concepts of thresholds of ischaemia in relation to brain structure and function. J Clin Pathol 30(Suppl 11):149_ 154

Villoslada P, Steinman L, Baranzini SE. (2009) Systems Biology and Its Application to the understanding of Neurological Diseases. Ann Neurol 65:124-139

Warach S. (2001) Tissue Viability Thresholds in Acute Stroke: The 4-Factor Model. Stroke 32:2460-2461

White BC, Sullivan JM, DeGracia DJ, O'Neil BJ, Neumar RW, Grossman LI, Rafols JA, Krause GS. (2000) Brain ischemia and reperfusion: molecular mechanisms of neuronal injury. J Neurol Sci 179(S 1-2):1-33

Yamada T, Bork P. (2009). Evolution of biomolecular networks: lessons from metabolic and protein interactions. Nat Rev Mol Cell Biol 10:791-803

Yenari M, Kitagawa K, Lyden P, Perez-Pinzon M. (2008) Metabolic downregulation: a key to successful neuroprotection? Stroke 39:2910-2917

Zhao H, Sapolsky RM, Steinberg GK. (2006) Phosphoinositide-3-kinase/akt survival signal pathways are implicated in neuronal survival after stroke. Mol Neurobiol $34: 249-270$ 